\title{
Haar Wavelet Based Implementation Method of the Non-integer Order Differentiation and its Application to Signal Enhancement
}

\author{
Yuanlu Li ${ }^{1,2}$, Chang $\operatorname{Pan}^{2}$, Xiao Meng ${ }^{2}$, Yaqing Ding ${ }^{2}$, Haixiu Chen ${ }^{1,2}$ \\ ${ }^{1}$ B-DAT, School of Information and Control, Nanjing University of Information Science \& Technology, \\ Nanjing, China, 210044; \\ ${ }^{2}$ Jiangsu Collaborative Innovation Center on Atmospheric Environment and Equipment Technology, \\ Nanjing University of Information Science \& Technology, Nanjing, China, 210044, yuanlu_li@163.com
}

\begin{abstract}
Non-integer order differentiation is changing application of traditional differentiation because it can achieve a continuous interpolation of the integer order differentiation. However, implementation of the non-integer order differentiation is much more complex than that of integer order differentiation. For this purpose, a Haar wavelet-based implementation method of non-integer order differentiation is proposed. The basic idea of the proposed method is to use the operational matrix to compute the noninteger order differentiation of a signal through expanding the signal by the Haar wavelets and constructing Haar wavelet operational matrix of the non-integer order differentiation. The effectiveness of the proposed method was verified by comparison of theoretical results and those obtained by another non-integer order differential filtering method. Finally, non-integer order differentiation was applied to enhance signal.
\end{abstract}

Keywords: Haar wavelet, non-integer order calculus, operational matrix, signal enhancement.

\section{INTRODUCTION}

$\mathrm{N}_{\mathrm{g}}^{\mathrm{o}}$ ON-INTEGER order differentiation (NIOD) is a generalization of the ordinary differentiation. Nowadays, NIOD attracts many scientists and engineers [1]. In fact, in the early eighties of the last century, semi-derivative voltammetry had been applied to peak resolution [2], and then theoretical and experimental resolution of semi-derivative linear scan voltammetry was further verified by Bobrowski et al. [3]. After the first application of the signal semi-differentiation, an entirely new perspective has been outlined in analytical science. Such as the fractional derivative was proposed to determine the overlapping band spectral parameters [4], the fractional order differentiation was used to improve signal resolution by Mocak et al. [5], the 2.5 order derivative combined with Fourier least square fitting was designed to process noised overlapped peaks [6], the fractional order derivative spectroscopy was used to resolve the overlapped Lorentzian peaks [7], the fractional order differentiation was widely used to detect the edges and enhance the texture of images [8]-[14]. In these applications, an important task is to implement the NIOD. A survey about the NIOD has been presented in [15], in which analysis, design and applications of analog and digital differentiators of fractional order were summarized.

At present, there are already some methods to implement the NIOD, these methods can be mainly divided into two categories: one is time-domain implementation methods of the NIOD, for example the most direct way is using the Grünwald-Letnikov definition to compute the NIOD of a signal, taking into account the non-locality of fractional differentiation, the short memory principle of the NIOD was proposed [16], in addition, there are implementations of the NIOD based Savitzky-Golay polynomial [17] and radial basis function [18]; the other is frequency-domain [19]-[23], such as fractional order FIR differentiators [19-22], and fractional order IIR differentiators [20, 23].

In this paper, a Haar wavelet-based implementation method of NIOD is proposed. In the proposed method, the operational matrices were used to compute the NIOD of a signal through expanding the signals by the Haar wavelets and constructing Haar wavelet operational matrix of the NIOD [7]. Advantage of the proposed method is that the NIOD of a signal can be easily obtained by matrix-vector multiplication.

In order to verify the effectiveness of the proposed method, some experiments have been performed to compare the proposed method with other methods [19, 23, 25]. Then the proposed method was used to enhance signal. In analytical chemistry and related sciences, because of the impact of environmental factors, the output signal is prone to drift and lead to an asymmetrical shape of peak, which will have effect on quantitative analysis. Ordinary derivative preprocessing technique $[26,27]$ or wavelet transform [28] was usually used to eliminate the effect of baseline drift before quantitative analysis. Here, we use the NIOD to enhance the signal. Processed result indicates that the result enhanced by the OFD is better than that processed by direct difference method (DM) and wavelet method (WM).

\section{HAAR WAVELET OPERATIONAL MATRIX OF THE NIOD}

\section{A. Fractional calculus}

The Riemann-Liouville definition is used in the following to generalize the Haar wavelet integral operational matrix from the integer order to the non-integer order.

$$
I^{r} x(t)=\frac{1}{\Gamma(r)} \int_{0}^{t} \frac{x(\tau)}{(t-\tau)^{1-r}} \mathrm{~d} \tau
$$


where $I^{r} x(t)$ denotes non-integer order integration (NIOI) of signal $x(t), \Gamma(r)$ is Gamma function and the order of the integration $r$ is an arbitrary real number.

\section{B. Haar wavelet and Haar wavelet expansion of a signal}

The Haar wavelets are defined as follows:

$$
h_{n}=h_{1}\left(2^{j} t-k\right), \quad n=2^{j}+k, \quad 0 \leq k \leq 2^{j}
$$

Where

$$
h_{0}(t)=1, \quad 0 \leq t<1, \quad h_{1}(t)= \begin{cases}1, & 0 \leq t<0.5, \\ -1, & 0.5 \leq t<1 .\end{cases}
$$

An arbitrary signal $x(t) \in L^{2}[0,1)$ can be expanded by Haar wavelet, i.e.,

$$
x(t)=\sum_{i=0}^{\infty} c_{i} h_{i}(t)
$$

where the Haar coefficients $c_{i}, i=0,1,2, \cdots$, are determined by

$$
c_{i}=2^{j} \int_{0}^{1} x(t) h_{i}(t) d t
$$

In practice, only the first $\mathrm{N}$ terms of (4) are considered, where $\mathrm{N}$ is a power of 2 . So we have

$$
x(t) \approx \sum_{i=0}^{N-1} c_{i} h_{i}(t)=C_{N}{ }^{T} H_{N}(t)=\hat{x}(t)
$$

where the superscript $T$ indicates transposition, the Haar coefficient vector $C_{N}$ and the Haar function vector $H_{N}(t)$ are defined as

$$
\begin{gathered}
C_{N} \triangleq\left[c_{0}, c_{1}, \ldots, c_{N-1}\right]^{T}, \\
H_{N}(t) \triangleq\left[h_{0}(t), h_{1}(t), \ldots, h_{N-1}(t)\right]^{T} .
\end{gathered}
$$

In order to obtain the coefficient vector $C_{N}$, we need $\mathrm{N}$ equations. So, collocation points are taken as

$$
t_{i}=\frac{(2 i-1)}{2 N}, \quad i=1,2, \ldots, N
$$

The $N$-square Haar matrix $\Psi_{N \times N}$ can be defined by

$$
\Psi \triangleq\left[H_{N}\left(\frac{1}{2 N}\right) \quad H_{N}\left(\frac{3}{2 N}\right) \quad \ldots \quad H_{N}\left(\frac{2 N-1}{2 N}\right)\right]
$$

C. Block pulse operational matrix of the non-integer order $\mathrm{N}$-term block pulse functions are defined as follows

$$
\varphi_{i}(t)=\left\{\begin{array}{ll}
1 & i T / N \leq t<(i+1) T / N \\
0 & \text { otherwise }
\end{array},\right.
$$

where $i=0,1,2, \cdots,(N-1)$.

This can be written in matrix form as

$$
\Phi_{N}(t)=\left[\varphi_{1}, \varphi_{2}, \cdots, \varphi_{N}\right]^{T}
$$

According to Ref. [24, 29], we have

$$
\left(I^{\alpha} \Phi_{N}\right)(t) \approx F^{\alpha} \Phi_{N}(t)
$$

where $F^{\alpha}$ is block pulse operational matrix for the noninteger order integration, and

$$
F^{\alpha}=\frac{1}{N^{\alpha}} \frac{1}{\Gamma(\alpha+2)}\left[\begin{array}{ccccc}
1 & \xi_{1} & \xi_{2} & \cdots & \xi_{N-1} \\
0 & 1 & \xi_{1} & \cdots & \xi_{N-2} \\
0 & 0 & 1 & \cdots & \xi_{N-3} \\
0 & 0 & 0 & \ddots & \vdots \\
0 & 0 & 0 & 0 & 1
\end{array}\right],
$$

with $\xi_{k}=(k+1)^{\alpha+1}-2 k^{\alpha+1}+(k-1)^{\alpha+1}$.

Let $D^{\alpha}$ be the block pulse operational matrix for the noninteger order differentiation. According to the property of fractional calculus, we have

$$
D^{\alpha} F^{\alpha}=I .
$$

From linear algebra we know that the inverse matrix of an upper triangular matrix is also upper triangular matrix, i.e.,

$$
D^{\alpha}=\left[F^{\alpha}\right]^{-1}=\left[\begin{array}{ccccc}
d_{0} & d_{1} & d_{2} & \cdots & d_{N-1} \\
0 & d_{0} & d_{1} & \cdots & d_{N-2} \\
0 & 0 & d_{0} & \cdots & d_{N-3} \\
0 & 0 & 0 & \ddots & \vdots \\
0 & 0 & 0 & 0 & d_{0}
\end{array}\right]
$$

Where

$$
d_{0}=1, \quad d_{1}=-\xi_{1} d_{0}, \quad \ldots, \quad d_{N-1}=-\sum_{k=1}^{N-1} \xi_{k} d_{N-k-1}
$$

D. Haar wavelet operational matrix of the non-integer order

Let

$$
\left(I^{\alpha} H_{N}\right)(t) \approx P_{N \times N}^{\alpha} H_{N}(t)
$$


where the N-square matrix $P_{N \times N}^{\alpha}$ is called the Haar wavelet operational matrix of the non-integer order integration.

Because the Haar wavelets are piecewise constant, then we have

$$
H_{N}=\Psi_{N \times N} \Phi_{N}
$$

From (17) and (18), we deduce

$$
\begin{aligned}
& \left(I^{\alpha} H_{N}\right)(t)=\left(I^{\alpha} \Psi_{N \times N} \Phi_{N}\right)(t) \\
& =\Psi_{N \times N}\left(I^{\alpha} \Phi_{N}\right)(t) \approx \Psi_{N \times N} F^{\alpha} \Phi_{N}(t)
\end{aligned}
$$

From (17) and (19), we obtain

$$
P_{N \times N}^{\alpha} H_{N}(t)=P_{N \times N}^{\alpha} \Psi_{N \times N} \Phi_{N}(t)=\Psi_{N \times N} F^{\alpha} \Phi_{N}(t)
$$

So, the Haar wavelet operational matrix of the non-integer order integration $P_{N \times N}^{\alpha}$ is given by

$$
P_{N \times N}^{\alpha}=\Psi_{N \times N} F^{\alpha} \Psi_{N \times N}^{-1}
$$

and the Haar wavelet operational matrix of the non-integer order differentiation $W_{N \times N}^{\alpha}$ is given by

$$
W_{N \times N}^{\alpha}=\Psi_{N \times N} D^{\alpha} \Psi_{N \times N}^{-1}
$$

\section{METHOD VALIDATION AND APPLICATION}

The proposed method is very easy. First, a signal is expanded with Haar wavelets according to (6). If the signal is noisy, we can set the threshold of the wavelet coefficients to reduce the noise of a signal. Second, the Haar wavelet operational matrix of the non-integer order differentiation $W_{N \times N}^{\alpha}$ is constructed by (22). Finally, the NIOD of a signal $D^{\alpha} x(t)$ can be approximated by $C_{N}{ }^{T} W_{N \times N}^{\alpha} H_{N}(t)$, i.e.

$$
D^{\alpha} f(t)=C_{N}{ }^{T} W_{N \times N}^{\alpha} H_{N}(t)
$$

In order to verify the proposed method, signal $x(t)=t$ is taken as an example to compare the proposed method with other methods $[19,23,25]$. This is because its fractional calculus can be calculated in theory, i.e.

$$
\left(I^{\alpha} x\right)(t)=\frac{\Gamma(2)}{\Gamma(2+\alpha)} t^{1+\alpha}, \text { and }\left(D^{\alpha} x\right)(t)=\frac{\Gamma(2)}{\Gamma(2-\alpha)} t^{1-\alpha}
$$

In interval $[0,1)$, we calculated non-integer order calculus of the signal using the proposed method at different $\alpha$, some results for $\alpha=0.2,0.5$ and 0.8 were shown in Fig.1. and Fig.2. We can see our result is in good agreement with the real result. Their maximum absolute errors for different $\mathrm{N}$ were given in Table 1. and Table 2.

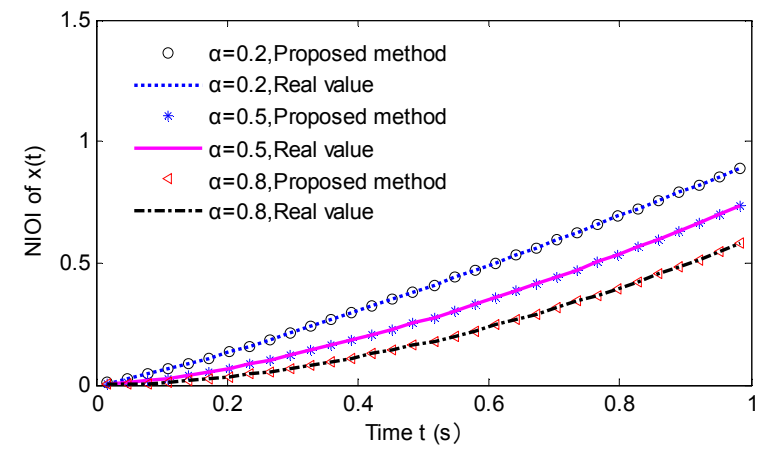

Fig.1. NIOI of $x(t)$.

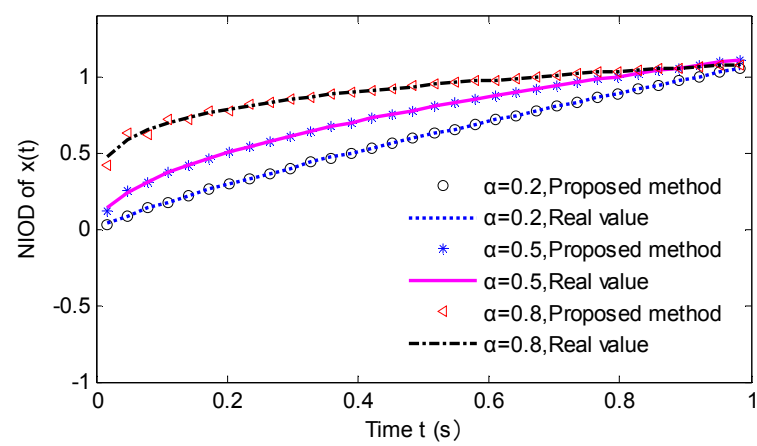

Fig.2. NIOD of $x(t)$.

Table 1. Maximum absolute errors for NIOI of $x(t)=t$ at different $\mathrm{N}$.

\begin{tabular}{|l|l|l|l|}
\hline & $\alpha=0.2$ & $\alpha=0.5$ & $\alpha=0.8$ \\
\hline $\mathrm{N}=32$ & $9.1788 \mathrm{e}-004$ & $6.0858 \mathrm{e}-004$ & $2.4794 \mathrm{e}-004$ \\
\hline $\mathrm{N}=64$ & $3.9953 \mathrm{e}-004$ & $2.1517 \mathrm{e}-004$ & $7.1203 \mathrm{e}-005$ \\
\hline $\mathrm{N}=128$ & $1.7391 \mathrm{e}-004$ & $7.6073 \mathrm{e}-005$ & $7.6294 \mathrm{e}-006$ \\
\hline
\end{tabular}

Table 2. Maximum absolute errors for NIOD of $x(t)=t$ at different $\mathrm{N}$.

\begin{tabular}{|l|l|l|l|}
\hline & $\alpha=0.2$ & $\alpha=0.5$ & $\alpha=0.8$ \\
\hline $\mathrm{N}=32$ & 0.0385 & 0.1410 & 0.4741 \\
\hline $\mathrm{N}=64$ & 0.0221 & 0.0997 & 0.4127 \\
\hline $\mathrm{N}=128$ & 0.0127 & 0.0705 & 0.3593 \\
\hline
\end{tabular}

From Table 1. and Table 2., one can see that their maximum absolute errors decrease with the increase of $\mathrm{N}$. Errors of NIOD are bigger than those of NIOI.

Further, we compared the proposed method with these methods in $[19,23,25]$. When $\alpha=0.5, \mathrm{~N}=32$, the comparison results for the non-integer order integration and differentiation are shown in Fig.3. and Fig.5. For the NIOI, one can see our result is in good agreement with the real result. From Fig.4. which is amplified parts of Fig.3., we can see our result is superior to that obtained with the methods in $[19,23,25]$. For the NIOD, one can also see our result is in good agreement with the real result. From Fig.6. which is amplified parts of Fig.5., we can see our result is superior to that obtained with the methods in [19, 23, 25]. 


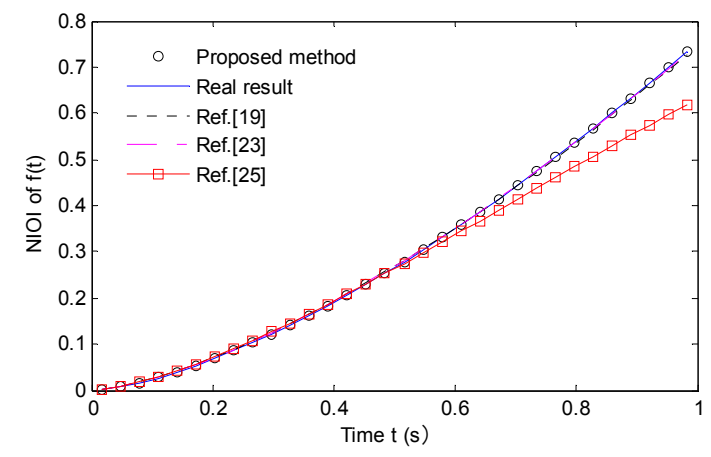

Fig.3. NIOI of $x(t)$ for different methods.

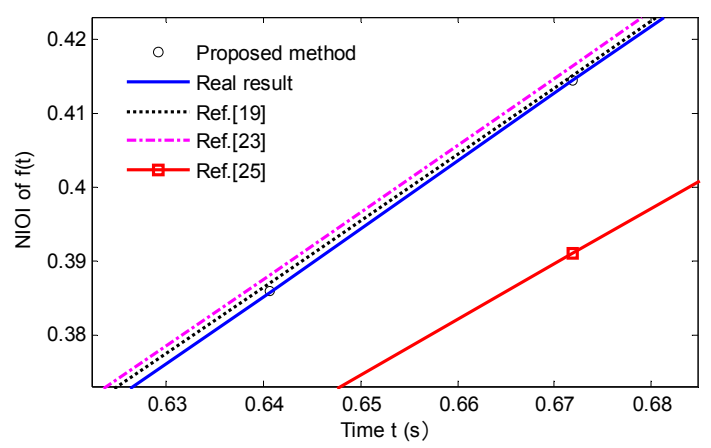

Fig.4. Magnified view of Fig.3.

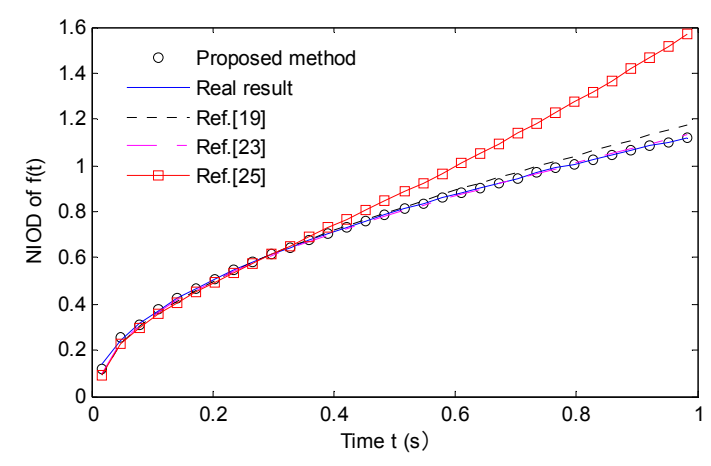

Fig.5. NIOD of $x(t)$ for different methods.

From (6), one knows if a signal with white noise is expanded by the Haar wavelets, we can de-noise by thresholding the wavelet coefficients. In the proposed method, one only needs to let the corresponding row of the Haar matrix be zeros. From [5], one knows that the differentiation or integration with fractional order (differintegration) is favorable mainly in the form of the semiintegral or semiderivative of the original measured signal. As a verification of the proposed method, we simulate a signal with baseline drift and white noise and compute its 0.5 order derivative using the proposed method and the G-L definition method. Their results are shown in Fig.7.b) and c). It is obvious that the proposed method has greater noise immunity than the G-L definition method.

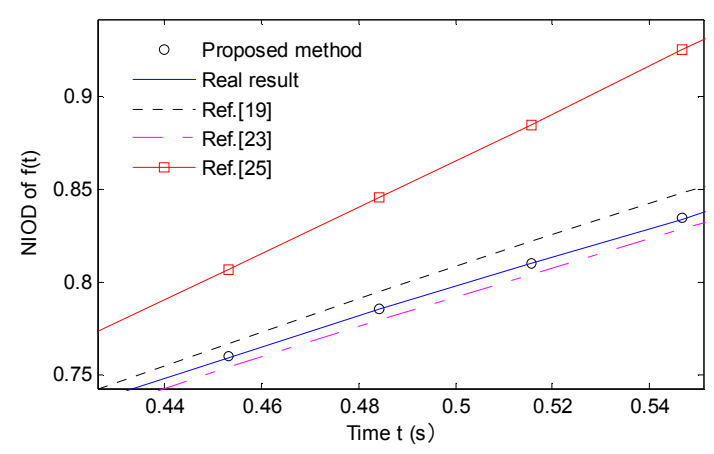

Fig.6. Magnified view of Fig.5.

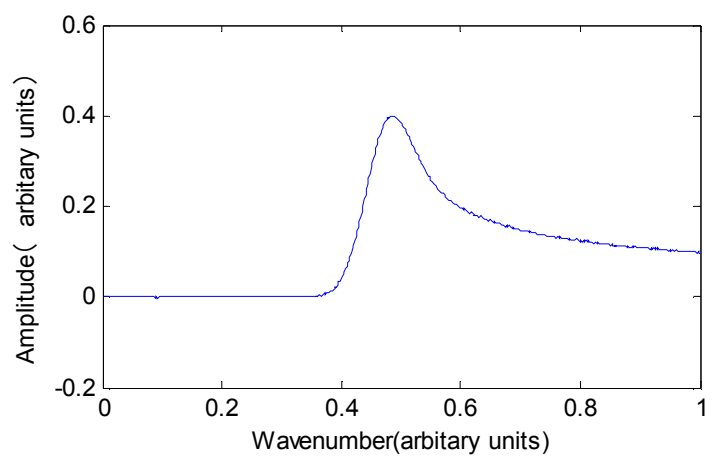

a). Original signal.

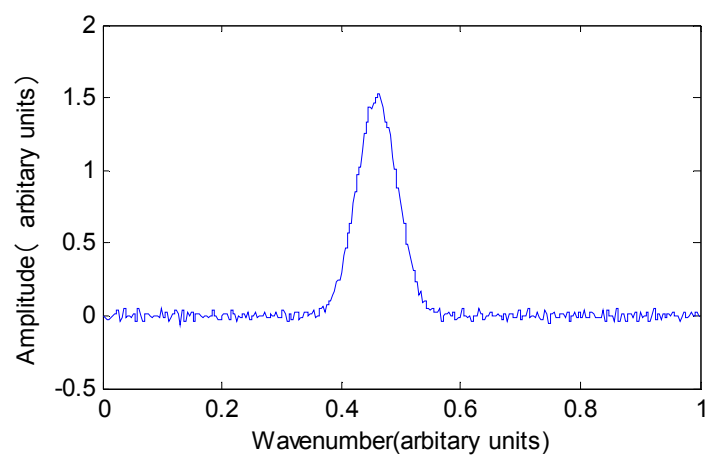

b). 0.5 order derivative of original signal obtained by the proposed method.

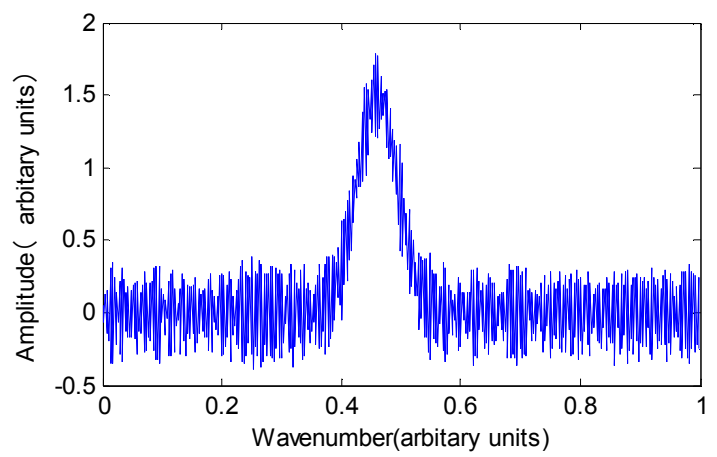

c). 0.5 order derivative of original signal directly obtained by the G-L definition method.

Fig.7. Original signal and its 0.5 order derivative. 


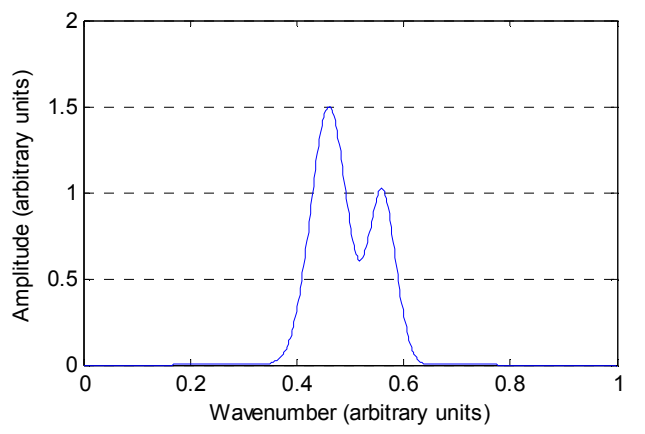

a). Normal signal.

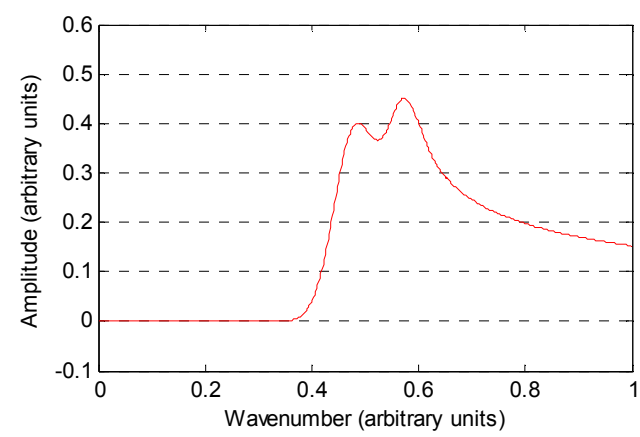

b). Original signal.

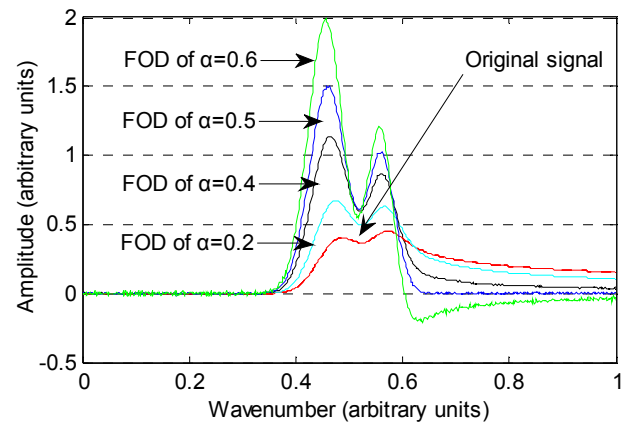

c). Enhancement results with NIOD.

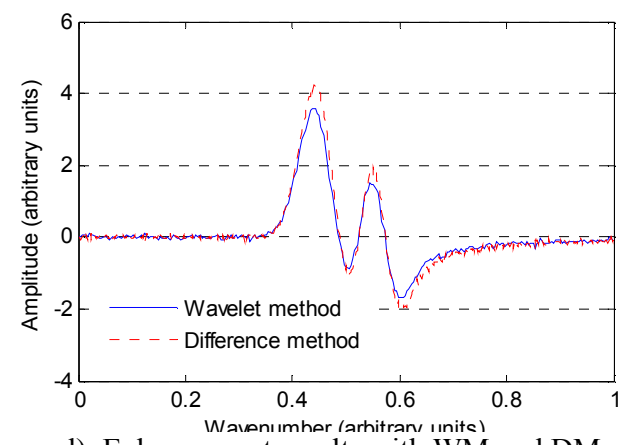

d). Enhancement results with WM and DM.

Fig.8. NIOD for signal enhancement and its comparison with WM and DM

In analytical chemistry and related sciences, because of the impact of environmental factors, the output signal is prone to drift and lead to an asymmetrical shape of peak, which will affect quantitative analysis. To eliminate the effect of baseline drift, Ordinary derivative preprocessing technique $[26,27]$ or wavelet transform [28] is the usual method. Here, we use the NIOD to enhance the signal. The signal shown in Fig.8.a) is a normal signal, and the signal in Fig.8.b) is an abnormal signal that has been drifted due to the impact of environmental factors or instrument. To eliminate the effect of baseline drift and enhance signal resolution, NIOD of the original signal was performed for different $\alpha$, and their results were shown in Fig.8.c). We can see that 0.5-order NIOD of the original signal can eliminate baseline drift and amplitude difference between two peaks. The shape of the signal enhanced with 0.5 -order NIOD is almost consistent with the normal signal in Fig.8.a).

Wavelet method (WM) and difference method (DM) are usually used to eliminate the effect of baseline drift and enhance signal resolution too. As a comparison, the results enhanced with wavelet method (WM) and difference method (DM) are shown in Fig.8.d). One can see that the enhancement results with WM and DM are consistent. But enhancement results with WM or DM are just a special case of the proposed method for $\alpha=1$.

It is obvious that using non-integer order differentiation to enhance the signal, we can obtain different enhancement results (Fig.8.c)). That is to say, non-integer order differentiation can provide a more flexible enhancing strategy.

\section{CONCLUSION}

The Haar wavelet-based implementation method of noninteger order differentiation is given and verified by comparison of theoretical results and those obtained by another non-integer order differential filtering method. Results indicate that the proposed method can not only implement non-integer order differentiation of a signal, but also reduce the effect of noise. In addition, because of the extension of differentiation orders from integer numbers to the fractional numbers, NIOD provides a more flexible enhancing strategy when we use the differential enhancement method. However, what are the general criteria to set order $\alpha$ is still an open problem.

\section{ACKNOWLEDGMENT}

The work was supported by National Natural Science Foundation of China (Grant: 61271395, 61302189).

\section{REFERENCES}

[1] Sabatier, J., Agrawal, O.P., Machado, J.T. (2007). Advances in Fractional Calculus. Springer.

[2] Toman, J.J., Brown, S.D. (1981). Peak resolution by semiderivative voltammetry. Analytical Chemistry, 53 (9), 1497-1504.

[3] Bobrowski, A., Kasprzyk, G., Mocák, J. (2000). Theoretical and experimental resolution of semiderivative linear scan voltammetry. Collection of Czechoslovak Chemical Communications, 65 (6), 979994.

[4] Kharintsev, S.S., Salakhov, M.K. (2004). A simple method to extract spectral parameters using fractional derivative spectrometry. Spectrochimica Acta Part A: Molecular and Biomolecular Spectroscopy, 60 (8), 2125-2133. 
[5] Mocak, J., Janiga, I., Rievaj, M., Bustin, D. (2007). The use of fractional differentiation or integration for signal improvement. Measurement Science Review, 7 (5), 39-42.

[6] Xiaoquan, L., Hongde, L., Min, Z., Xiaoqiang, W., Zhonghua, X., Jingwan, K. (2003). Fractional derivative combined fourier-least square fitting to process noised overlapped signal. Chinese Journal of Analytical Chemistry, 31 (2), 143-147.

[7] Li, Y.L., Tang, H.Q., Chen, H.X. (2011). Fractionalorder derivative spectroscopy for resolving simulated overlapped Lorenztian peaks. Chemometrics and Intelligent Laboratory Systems, 107 (1), 83-89.

[8] Pu, Y., Wang, W., Zhou, J., Wang, Y., Jia, H. (2008). Fractional differential approach to detecting textural features of digital image and its fractional differential filter implementation. Science in China Series F: Information Sciences, 51 (9), 1319-1339.

[9] Mathieu, B., Melchior, P., Oustaloup, A., Ceyral, C. (2003). Fractional differentiation for edge detection. Signal Processing, 83 (11), 2421-2432.

[10] Gao, C., Zhou, J., Zheng, X., Lang, F. (2011). Image enhancement based on improved fractional differentiation. Journal of Computational Information Systems, 7 (1), 257-264.

[11] Gao, C.B., Zhou, J.L., Hu, J.R., Lang, F.N. (2011). Edge detection of colour image based on quaternion fractional differential. IET Image Processing, 5 (3), 261-272.

[12] Cuesta, E., Kirane, M., Malik, S.A. (2012). Image structure preserving denoising using generalized fractional time integrals. Signal Processing, 92 (2), 553-563.

[13] Ren, Z., He, C., Zhang, Q. (2013). Fractional order total variation regularization for image superresolution. Signal Processing, 93 (9), 2408-2421.

[14] Khanna, S., Chandrasekaran, V. (2012). Fractional derivative filter for image contrast enhancement with order prediction. In IET Conference on Image Processing (IPR 2012). IEEE, 1-6.

[15] Krishna, B.T. (2011). Studies on fractional order differentiators and integrators: A survey. Signal Processing, 91 (3), 386-426.

[16] Podlubny, I. (1998). Fractional Differential Equations: An Introduction to Fractional Derivatives, Fractional Differential Equations, to Methods of Their Solution and Some of Their Applications (Vol. 198). Academic Press.
[17] Chen, D., Chen, Y.Q., Xue, D. (2011). Digital fractional order Savitzky-Golay differentiator. IEEE Transactions on Circuits and Systems II: Express Briefs, 58 (11), 758-762.

[18] Tseng, C.C., Lee, S.L. (2010). Design of fractional order digital differentiator using radial basis function. IEEE Transactions on Circuits and Systems I: Regular Papers, 57 (7), 1708-1718.

[19] Tseng, C.C. (2001). Design of fractional order digital FIR differentiators. IEEE Signal Processing Letters, 8 (3), 77-79.

[20] Tseng, C.C. (2007). Design of FIR and IIR fractional order Simpson digital integrators. Signal Processing, 87 (5), 1045-1057.

[21] Tseng, C.C., Pei, S.C., Hsia, S.C. (2000). Computation of fractional derivatives using Fourier transform and digital FIR differentiator. Signal Processing, 80 (1), 151-159.

[22] Tseng, C.C. (2006). Design of variable and adaptive fractional order FIR differentiators. Signal Processing, 86 (10), 2554-2566.

[23] Chen, Y., Vinagre, B.M. (2003). A new IIR-type digital fractional order differentiator. Signal Processing, 83 (11), 2359-2365.

[24] Li, Y., Sun, N. (2011). Numerical solution of fractional differential equations using the generalized block pulse operational matrix. Computers \& Mathematics with Applications, 62 (3), 1046-1054.

[25] Ferdi, Y. (2009). Impulse invariance-based method for the computation of fractional integral of order $0<\alpha<1$. Computers \& Electrical Engineering, 35 (5), 722-729.

[26] Griffiths, T.R., King, K., Hubbard, H.V.S.A., Schwing-Weill, M.J., Meullemeestre, J. (1982). Some aspects of the scope and limitations of derivative spectroscopy. Analytica Chimica Acta, 143, 163-176.

[27] O'Haver, T.C., Fell, A.F., Smith, G. et al. (1982). Derivative spectroscopy and its applications in analysis. In Analytical Proceeding, 19 (1), 22-46.

[28] Shao, X., Cai, W., Sun, P., Zhang, M., Zhao, G. (1997). Quantitative determination of the components in overlapping chromatographic peaks using wavelet transform. Analytical Chemistry, 69 (9), 1722-1725.

[29] Chi-Hsu, W. (1983). On the generalization of block pulse operational matrices for fractional and operational calculus. Journal of the Franklin Institute, 315 (2), 91-102.

Received November 18, 2014. Accepted June 18, 2015. 